\section{Tamoxifen erythroid toxicity revealed by studying the role of nuclear receptor co-activator 4 in erythropoiesis}

We read with great interest the paper recently published by Santana-Codina et al. ${ }^{1}$ about the cell autonomous and non-autonomous role of nuclear receptor co-activator 4 (NCOA4). NCOA4 is a cargo receptor that, in conditions of iron deficiency, promotes ferritinophagy to release iron from ferritin. ${ }^{2,3}$ Inactivation of Ncoa4 in C57BL/6 mice causes mild microcytic anemia and increases the susceptibility to iron-deficiency anemia due to iron being trapped in ferritin in several organs. ${ }^{3,4}$ To formally prove the role of Ncoa4 inactivation on erythropoiesis, a tamoxifen-inducible CRE-dependent Ncoa4-null model was generated. ${ }^{1}$ Five days of tamoxifen treatment induced Ncoa4 depletion in adult Ncoa4-floxed mice $\left(\mathrm{Ncoa}_{4}^{\mathrm{rec}}\right)$. Seven days after tamoxifen discontinuation, a rapid and transient normochromic normocytic anemia was observed (corresponding to day 11 from the first tamoxifen injection); recovery started 14 days after drug interruption (corresponding to day 18 from the first tamoxifen injection). ${ }^{1}$ Based on these results, the Authors conclude that NCOA4 is essential for acute erythropoiesis expansion in adult mice, as in germ-line Ncoa4-knockout (ko) neonates who develop severe postnatal anemia. ${ }^{5}$

We were puzzled by the rapid development of anemia and the prompt recovery in tamoxifen-treated animals, and wondered whether such an effect could be related to tamoxifen toxicity on Ncoa4 null erythroid cells. To address this point, we challenged adult germ-line Ncoa4-ko mice with tamoxifen, using the same protocol as in Santana-Codina et al. ${ }^{1}$ Briefly, 12 -week old Sv129/J Ncoa4-ko and wild-type littermates received $200 \mathrm{mg} / \mathrm{kg}$ tamoxifen via oral gavage daily for five consecutive days (day 0-4) and complete blood count was obtained at days 0, 4, 11 and 21. We chose mice on Sv129/J background that, unlike C57BL/6 Ncoa4-ko animals, ${ }^{4}$ do not show anemia or alterations of iron parameters but only mild microcytosis (Figure 1 and Nai et al., 2019, manuscript in preparation). At day 4, only Ncoa4-ko mice showed a statistically significant decrease in red blood cell (RBC) count, and hematocrit (Hct) and hemoglobin ( $\mathrm{Hb})$ levels. At day 11, also wild-type mice showed a reduction in $\mathrm{RBC}$ count and decreased $\mathrm{Hb}$ and Hct, although for the latter two parameters levels were higher than those of Ncoa4-ko mice. At day 21, hematologic parameters started to recover in both genotypes. Mean corpuscular volume and mean corpuscular hemoglobin, low in germ-line Ncoa4-ko mice, were not significantly altered (Figure 1). The tamoxifen effect on erythropoiesis was not strainrelated, since it was also observed in C57BL/6 wild-type mice (Online Supplementary Table S1). Thus, acute tamoxifen treatment, commonly used to induce gene inactivation in CRE transgenic mice, may decrease $\mathrm{RBC}, \mathrm{Hb}$ and Hct in wild-type animals, with a quick recovery upon drug discontinuation. The effect was stronger in Ncoa4ko animals, suggesting greater sensitivity to the drug.

A similar bias likely affects the phenylhydrazine (PHZ)based experiments performed by Santana-Codina et al. to induce hemolysis during tamoxifen administration. ${ }^{1}$ It is reasonable to suppose that the more severe anemia developed by $N \mathrm{Noa}_{4}^{\mathrm{rec}}$ mice results from the hypersensitivity of Ncoa4-null erythroid cells to tamoxifen toxicity, rather than from increased hemolysis. In support of this
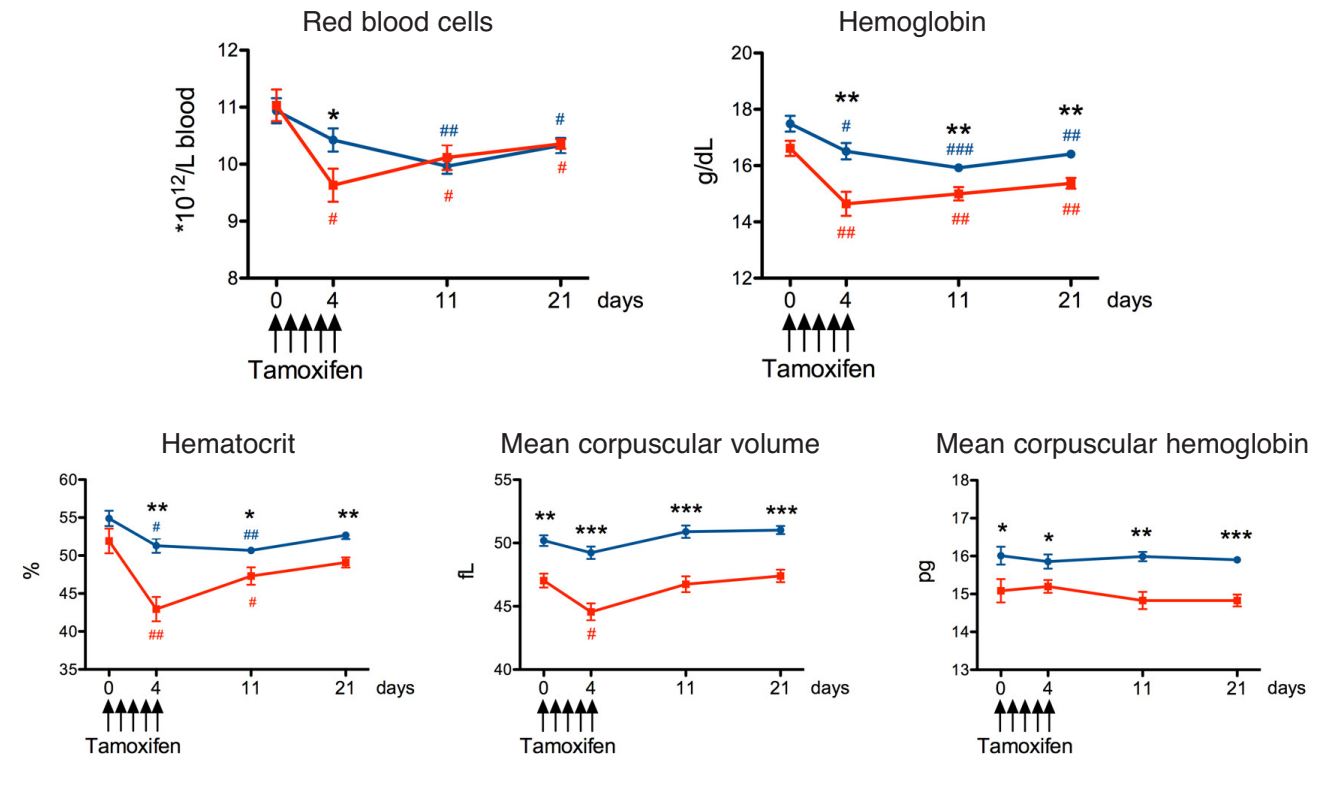

$\rightarrow$ wild-type $\rightarrow$ Ncoa4-ko

Figure 1. Hematologic parameters of Ncoa4-ko mice treated with tamoxifen. Ten-week old Sv129/J Ncoa4-knockout (ko) mice and wild-type (wt) littermates, both males (5 wt and 4 Ncoa4-ko) and females ( 5 wt and 3 Ncoa4-ko), were administered $200 \mathrm{mg} / \mathrm{kg}$ tamoxifen (Sigma-Aldrich, T56548, $20 \mathrm{mg} / \mathrm{mL}$ in corn oil) via oral gavage daily for five consecutive days (day 0-4). Complete blood count was determined at days $0,4,11$ and 21 . Error bars indicate Standard Error. Statistically significant differences between Ncoa4-ko and wt mice at each time point: $* P<0.05 ; * \star P<0.01 ; * * * P<0.005$. Statistically significant differences versus untreated mice of each genotype at each time point: ${ }^{\#} P<0.05 ; " \# P<0.01 ;{ }^{\# \# \#} P<0.005$. 
hypothesis, after $\mathrm{PHZ}$ administration, anemia was similar in mice with constitutive erythroid Ncoa4 deletion and in controls. ${ }^{1}$ The Authors claim that this discrepancy might be due to a NCOA4 effect in non-erythroid tissues; however, to formally prove this assumption, Ncoa $4^{\text {rec }}$ mice should have been treated with PHZ at later time points, i.e. after recovery from tamoxifen toxicity.

NCOA4, originally identified as a co-activator of the androgen receptor, was shown to regulate the activity of additional nuclear receptors, including the estrogen receptors. ${ }^{6}$ Estrogen modulates erythroid differentiation in a variety of ways ${ }^{7,8}$ and tamoxifen, as a selective estrogen receptor modulator (SERM), can behave as an estrogen agonist or antagonist, depending on the tissue-specific balance among co-activators and repressors. ${ }^{9}$ Of note, patients treated with tamoxifen may develop anemia as a side effect (1-10\% of cases; Apotex Inc., Toronto, Ontario, Canada. Product monograph. APO-TAMOX. Control number: 201525. Date of revision: Feb 19 2018). Several other nuclear receptors, such as peroxisome proliferator-activated receptor $\alpha$, glucocorticoid receptor and thyroid hormone receptor $\beta$, whose activity is influenced by NCOA4, regulate erythroid progenitor self-renewal or terminal differentiation. ${ }^{5,10}$ Thus, it is not surprising that the genetic ablation of NCOA4 enhances the toxicity of tamoxifen on erythroid cells. Further studies are required to clarify the mechanisms of tamoxifen toxicity on the erythroid compartment and of the increased susceptibility of Ncoa4-null cells. However, our findings question an essential role for NCOA4 in erythropoiesis, considering the acute anemia described in Ncoa $4^{\text {rec }}$ mice $^{1}$ to be the result of tamoxifen toxicity rather than of Ncoa4 deletion per se. The real effect of Ncoa 4 deficiency in Ncoa $4^{\text {rec }}$ mice becomes evident at a later time point (day 46), when also microcytosis develops (see Figure 2 in Santana-Codina et al. ${ }^{1}$ ), probably secondary to iron sequestration into ferritin and iron restricted erythropoiesis.

In conclusion, our results uncover a previously unrecognized suppressive effect of tamoxifen on erythroid cells, the severity of which is increased by the loss of NCOA4. As a consequence, NCOA4 function in the setting of acute erythropoietic expansion and increased iron demand needs to be reconsidered. In addition, in murine models featuring tamoxifen-induced Cre-dependent gene ablation, tamoxifen toxicity should always be monitored, and hematologic data in the 14 days following tamoxifen treatment should be interpreted with caution.

Antonella Nai, ${ }^{1,2}$ Mariateresa Pettinato, ${ }^{1,2}$ Giorgia Federico, ${ }^{3}$ Violante Olivari, ${ }^{1}$ Francesca Carlomagno ${ }^{3^{*}}$ and Laura Silvestri, ${ }^{1,2^{*}}$

${ }^{*}$ Contributed equally as co-senior authors
'Division of Genetics and Cell Biology, Ospedale San Raffaele, Milan; ${ }^{2}$ Vita-Salute San Raffaele University, Milan and ${ }^{3}$ Department of Molecular Medicine and Medicine Biotechnology (DMMBM), University of Naples Federico II, Institute of Endocrinology and Experimental Oncology (IEOS), CNR, Naples, Italy

Correspondence: FRANCESCA CARLOMAGNO

francesca.carlomagno@unina.it

\section{LAURA SILVESTRI}

silvestrilaura@hsr.it

doi:10.3324/haematol.2019.224857

Information on authorship, contributions, and financial \& other disclosures was provided by the authors and is available with the online version of this article at www. haematologica.org.

\section{References}

1. Santana-Codina N, Gableske S, Quiles Del Rey M, et al. NCOA4 maintains murine erythropoiesis via cell autonomous and nonautonomous mechanisms. Haematologica. 2019 Jan 10. [Epub ahead of print]

2. Mancias JD, Wang X, Gygi SP, Harper JW, Kimmelman AC. Quantitative proteomics identifies NCOA4 as the cargo receptor mediating ferritinophagy. Nature. 2014;509(7498):105-109.

3. Dowdle WE, Nyfeler B, Nagel J, et al. Selective VPS34 inhibitor blocks autophagy and uncovers a role for NCOA4 in ferritin degradation and iron homeostasis in vivo. Nat Cell Biol. 2014;16(11):10691079.

4. Bellelli R, Federico G, Matte A, et al. NCOA4 Deficiency Impairs Systemic Iron Homeostasis. Cell Rep. 2016;14(3):411-421.

5. Gao X, Lee HY, Li W, et al. Thyroid hormone receptor beta and NCOA4 regulate terminal erythrocyte differentiation. Proc Natl Acad Sci U S A. 2017;114(38):10107-10112.

6. Kollara A, Brown TJ. Expression and function of nuclear receptor coactivator 4: evidence of a potential role independent of co-activator activity. Cell Mol Life Sci. 2012;69(23):3895-3909.

7. Blobel GA, Orkin SH. Estrogen-induced apoptosis by inhibition of the erythroid transcription factor GATA-1. Mol Cell Biol. 1996;16(4):1687-1694.

8. Kim HR, Lee JH, Heo HR, et al. Improved hematopoietic differentiation of human pluripotent stem cells via estrogen receptor signaling pathway. Cell Biosci. 2016;6(1):50.

9. Jordan VC. Selective estrogen receptor modulation: concept and consequences in cancer. Cancer Cell. 2004:5(3):207-213.

10. Lee HY, Gao X, Barrasa MI, et al. PPAR-alpha and glucocorticoid receptor synergize to promote erythroid progenitor self-renewal. Nature. 2015;522(7557):474-477. 